\title{
Opensensor - An open wireless sensor platform
}

\author{
Anders Grauballe \\ Aalborg University \\ Dept. of Electronic Systems \\ Aalborg - Denmark \\ agraubal@es.aau.dk
}

\author{
Gian Paolo Perrucci \\ Aalborg University \\ Dept. of Electronic Systems \\ Aalborg - Denmark \\ gpp@es.aau.dk
}

\author{
Frank H.P. Fitzek \\ Aalborg University \\ Dept. of Electronic Systems \\ Aalborg - Denmark \\ ff@es.aau.dk
}

\begin{abstract}
This paper introduces the opensensor platform. The idea behind the opensensor is to build an open source wireless sensor platform. All relevant information to build, to program and to teach the opensensor is freely available. The opensensor platform can be used for all kind of wireless sensor network research, but in this paper the focus is on contextual information retrieval for the mobile phone. After introducing and motivating the opensensor platform, some first examples and projects for contextual information retrieval are introduced.
\end{abstract}

\section{Categories and Subject Descriptors}

B.7 [Integrated circuits]: Miscellaneous

\section{General Terms}

contextual information retrieval for mobile phones

\section{Keywords}

Mobile phones, wireless sensor network, open source

\section{GENERAL INTRODUCTION}

In a very short period of time mobile phones have penetrated our daily life after their introduction in the late 80's. Undoubtedly, mobile phones have changed our life. The very first service of mobile phones combined mobility and voice. Throughout the last decades further services, in addition to voice services, have been added. Nowadays, mobile phones host a large number of services and features for the user such as Internet access, digital camera, mp3 player and many more. Despite the fact that there are many services in the mobile phone already now and more on the way, mobile phones have nearly no knowledge about the user or its surrounding. Such knowledge, also referred to as cognition or contextual information, however would help to create new services or extend the existing ones. One example for the

Mobimedia 2008 July 7-9, 2008, Oulu, Finland.

Copyright 2008 ICST ISBN

978-963-9799-25-7/08/07

DOI 10.4108/ICST.MOBIMEDIA2008.4024 extension of existing services could be that the mobile phone recognizes the type of scenario (or location) it is operating, e.g., whether the user is in the bathroom or within an airplane and based on that acts accordingly. For instance, the mobile phone should not accept any phone calls or switch off entirely, respectively.

Parallel with the evolution of the mobile phone, wireless sensor networks (WSN) have attracted a lot of attention. The initial research was mainly focused on military applications, but wireless sensors have shown their applicability to civil environments too. A wireless sensor is composed out of a sensory part, a wireless communication facility and a battery. The sensor measures some physical parameters such as light, temperature, or distance. There are also some passive sensors such as RFID and UHF devices that do not need a battery and are empowered by the electromagnetic waves from the requesting device. But in this paper we will focus on active sensors with batteries. Wireless sensors have great capabilities to retrieve contextual information.

The combination of mobile phones with wireless sensors is quite obvious and straight forward. Today there exist two main ways of this combination, namely embedded and external sensors. Embedded sensors are part of the mobile phone and can be accessed through the internal APIs of the mobile phone. For instance, Apple is using an accelerometer on the iPhone to retrieve information whether the user is operating the device in portrait or landscape mode. Such accelerometers can be found in many more devices already and used for different kind of applications. Paul Coulton [1] has launched some master pieces using the accelerometer for mobile games on a Nokia N95 device. Before the iPhone was even launched, Nokia came up with their model 5500. This device was intended for joggers counting the food steps. Besides those first applications of embedded sensors, new sensors are on their way. Note that some other sensors are already on the devices but not necessarily recognized by everybody as sensors, such as the microphone, the camera, the GPS module. The use of the GPS module as a sensor was shown by Christian Søndergaard Jensen [2]. By combing the GPS location of several taxis in the city of Aalborg, Denmark, the traffic flow within the city was derived.

Besides the embedded sensors, external sensors are also used. Apple and Nike offer an external sensor to be placed into the shoe conveying the number of steps to the iPod. Other sensors are capable to retrieve heart beats or blood pressure and convey that information to a dedicated place such as the watch or the mobile device. It is expected that the variety of external sensors is much larger than those of the embedded 
ones. Especially the external sensor will lead to new applications in the area of health care, intelligent housing and others. The advantage of omnipresent sensors is that whole measurement campaigns can be carried out, which are more meaningful than instant measurements points. An example is health parameters such as blood pressure or insulin levels. In the future more external sensors will be on the market such that the mobile device will be surrounded by many sensors with different tasks. Furthermore the architecture of external sensors may change. Besides pure forwarding of the sensed data, a new generation of sensors will emerge, preprocessing the sensor data before conveying it to the mobile phone. The combination of external sensors and the mobile phone is a logical step as the mobile phone has sophisticated display functionality for human machine interaction. Furthermore the mobile phone is one dedicated sink where the user expects the information to be collected, as given in Figure 1. The figure shows 11 sensors of different classes. One class may be used for temperature measurements, while a second could be sensing light values and a third could sense humidity. The result of the measurement is made visible on the mobile device. Despite the displaying function, the mobile device (MD) is responsible for data fusion.

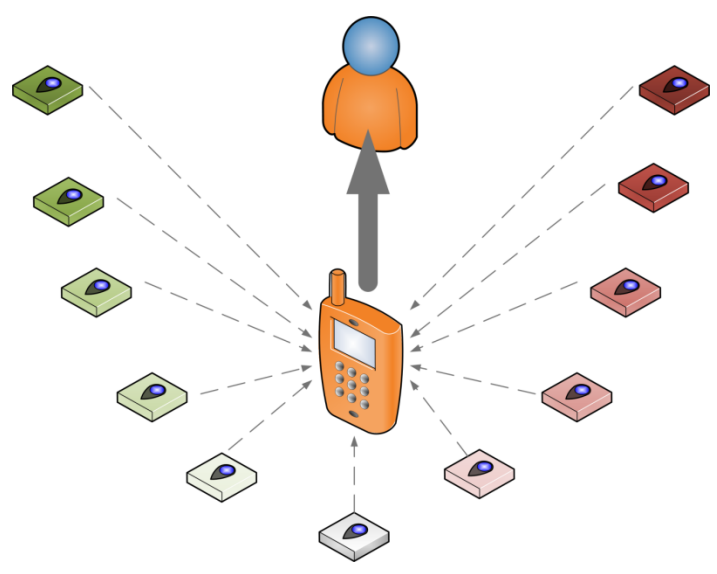

Figure 1: Different classes of external sensors conveying information to the user through the mobile phone.

However, embedded or external sensors, both will provide the mobile device with contextual information of and for the user. In the following we will introduce the opensensor as one possible collector for such contextual information. By means of several project examples the idea of contextual information retrieval is presented and discussed.

\section{OPENSENSOR PLATFORM}

In the following we will introduce our opensensor platform. We will advocate the need for such a platform and introduce the individual open parts.

\subsection{Motivation for developing the opensensor platform}

There are already some sensor platforms on the market. Nevertheless, in collaboration with the Technical University of Berlin, the mobile device group of Aalborg University (AAU) [3] has decided to design their own platform. The main reason was to have as much flexibility on the platform as possible. Flexibility refers to the number of connectable sensor parts as well as the wireless part. As the opensensor should be used for ongoing and future research projects as well as for teaching purposes, the design of the board was kept as simple as possible. In order to facilitate implementation of sensor boards surface-mount devices (SMD) technology was not considered. This, the opensensor employs easy-to mount standard components (e.g, through-the-hole), resulting in a very low-cost solution that can openly and easily implemented by researchers and students. As the mobile device group of AAU has a huge interest in the convergence of wireless sensors with mobile phones, the opensensor offers the possibility to communicate with commercial mobile phones. As most third party solutions for wireless networking are closed solutions, we decided to make the whole opensensor concept freely available for everybody [4]. Not just open source, but also open hardware and open teaching material. We hope to inspire other researchers to use our platform and eventually contribute to the project with more software or teaching material.

\section{2 opensensor hardware}

The opensensor platform is shown in Figure 2. It is powered up with a $9 \mathrm{~V}$ battery block. The board has several interfaces to connect to the outer world. Two LEDs are on the board. The red LED indicates the operational phase of the opensensor while the green LED can be controlled from the DSP and is used for monitoring purposes. The main parts of the opensensor are introduced in the following.

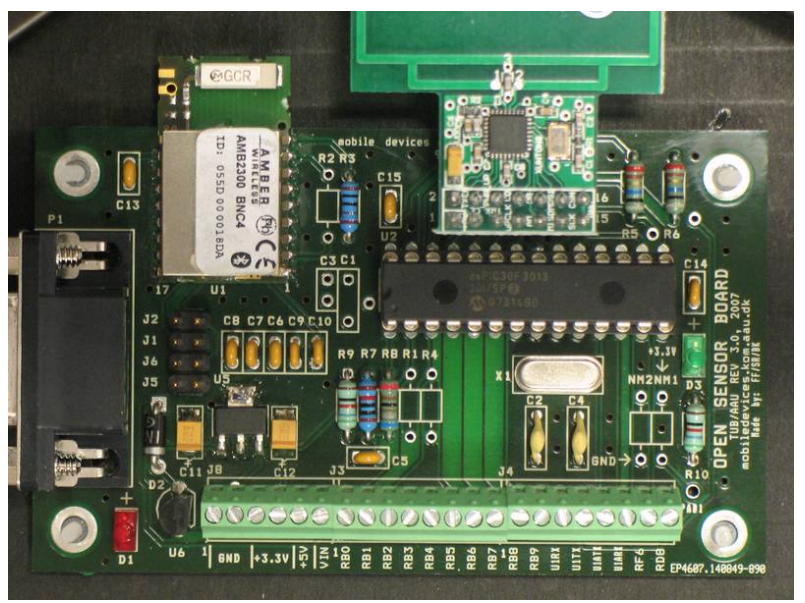

Figure 2: A picture of the opensensor board (version $3.0)$.

\subsubsection{DSP}

The core of the opensensor is a 16 bit architecture dsPIC$30 f 3013$ processor produced by Microchip. It has low power consumption, features $24 \mathrm{~KB}$ of program memory, $2.048 \mathrm{~B}$ of RAM and has 28 pins of where 20 can be used as I/O ports. For communication purposes the chip has two UARTS and one SPI port. The chip is capable of performing Analog-to Digital conversion in 12 bit, 200Ksps for measurement pur- 
poses, has three timers and is able to provide pulse-width modulation (PWM) which can be utilized for motor controlling. It is possible to program the chip when it is integrated into a circuit, which makes the it ideal for development purposes such as the opensensor.

\subsubsection{Communication and Programming Interfaces}

The opensensor board has up to five interfaces that allow communicating and programming the sensor. While the connection bar, the RS232 and the PICKITPINS are part of the board, the wireless communication interfaces nRF905 and Bluetooth are optional.

\section{RS232.}

A RS232 interface connected to one of the UARTs on the dsPIC30f3013. This RS232 interface can be used to connect to the PC. Prior opensensor boards used mini USB for data exchange but also for powering the board while programming on it. The choice for an RS232 was based on the costs related to the mini USB port and its needed additional hardware.

\section{nRF905.}

The nRF905 is a radio interface without any link layer or medium access protocol. The frequencies which the interface can use is 433, 868 and $915 \mathrm{MHz}$. The interface is connected to the SPI interface of the dsPIC30f3013 and enables the opensensor to form WSNs, as well as exchanging data in this WSN using a protocol defined by the developer. A basic CSMA/CA protocol is available on the opensensor web page [4], but the medium access scheme can be changed according to the needs of the project or just for educational purposes. Each packet is broadcasted on the air and can be received by all other nodes. The addressing of the packets is done by the framing as given in [5].

\section{Bluetooth.}

As most commercial phones support Bluetooth, the opensensor has the option to add a Bluetooth chip. The current version 3.0 of the opensensor hosts a chip from Amber Wireless connected to the the other UART of the dsPIC30f3013. As the Bluetooth chip itself costs more than the whole opensensor platform, it should be evaluated beforehand if the Bluetooth is needed on all sensor boards. In many of our examples, most sensor boards communicate over the nRF905 interface with each other and one dedicated sensor, equipped with Bluetooth connectivity, conveys the results to the mobile phone.

\section{Programmer Pin.}

The opensensor can be programmed through the programmer pin where the PICkit2 can be connected. The PICkit2 programmer must be connected to the USB interface of the $\mathrm{PC}$ and is able to operate in both Windows and Linux environments.

\section{Connection Bar.}

The basic version of the opensensor does not host any real sensor part. But any sensor can be attached to the opensensor easily. Here we explain shortly how to connect a light sensor. The light sensor must be mounted to one of the input pins as in Figure 3. It is then possible to use the

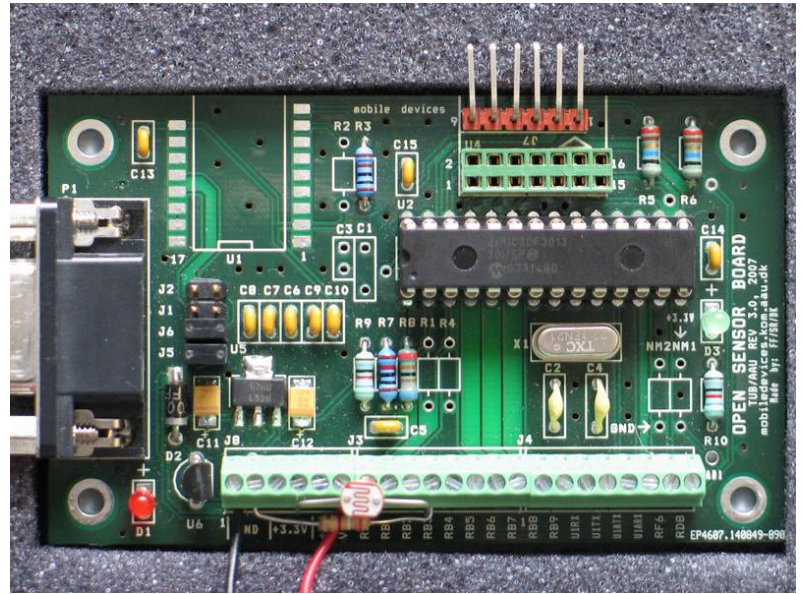

Figure 3: The light sensor hardware is mounted on one of the input ports of the opensensor.

Analog-to-Digital converter in the opensensor to measure the level of light. The light sensor can be replaced with any other sensor such as for temperature or distance. The software to retrieve the light values can be found on the web page [4] or seen in Figure 4.
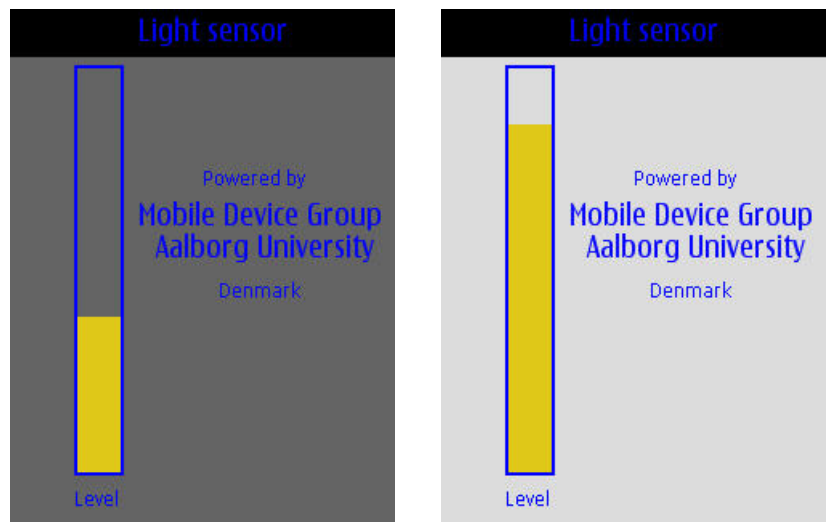

Figure 4: Screenshots from the software that allows the user to see the light level using a light sensor.

\section{3 opensensor Software}

\subsubsection{Programming Environment}

In the following we explain shortly how to use the MPLAB Integrated Development Environment (IDE) for programming the opensensor. Most of the examples on our web page use Windows as operating system for the PC. Nevertheless in [4] the Linux support for the opensensor project is described as well.

\section{MPLAB.}

MPLAB is the programming environment used to develop programs for the opensensor. Both $\mathrm{C}$ and Assembler code can be used according to the programmer's skills. It is possible to setup projects and build them using the GUI interface as shown in Figure 5. 


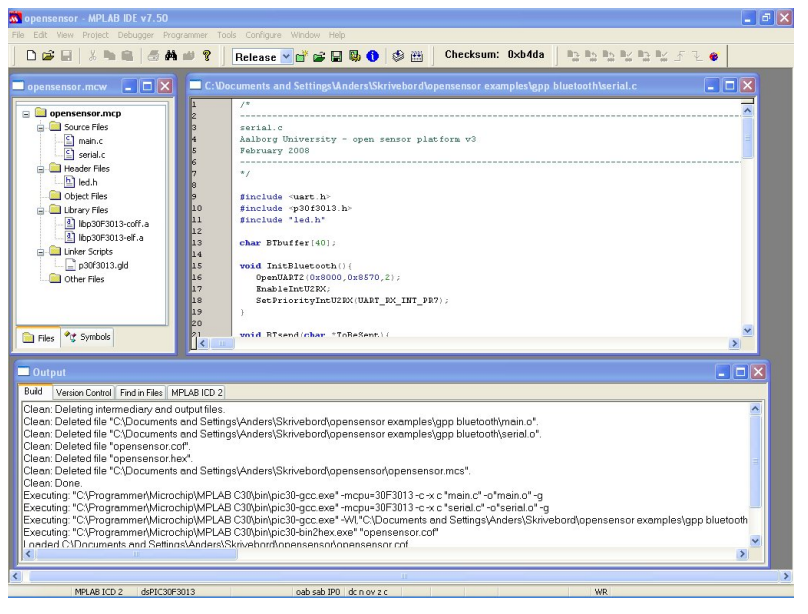

Figure 5: A screenshot of MPLAB IDE.

\section{Console.}

The opensensor can be accessed through the RS232 / Bluetooth interface via a terminal program. This makes debugging on the opensensor easier, because simple text commands can test various programmed functions. An example of the console can be seen in Figure 6 where commands are used to turn the green LED on or off.

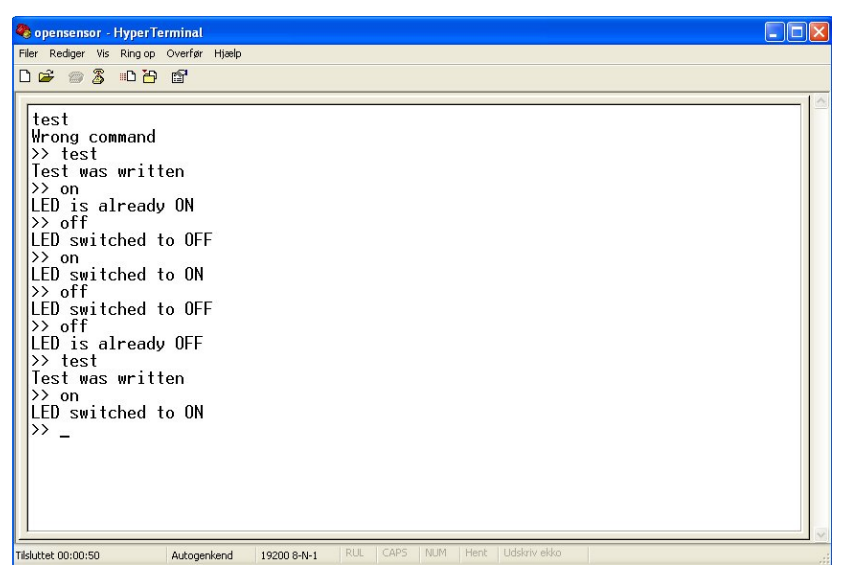

Figure 6: A screenshot of the Windows Hyper Terminal that allows sending and receiving data to/from the opensensor through the RS232 or Bluetooth interface for debugging or testing purposes.

\subsection{Mobile phone software}

The opensensor platform allows commercial mobile phones to interact with it using Bluetooth. This communication is based on the RFCOMM protocol which is a simple set of transport protocols, made on top of the L2CAP, providing an RS-232 serial port emulation. There are two approaches to describe the interaction between the opensensor an the mobile phone: the gateway approach and the retrieval approach. In the first one, the opensensor uses the mobile phone as a gateway to send information to the outer world or directly to the user. For example, if a temperature sensor is mounted on the board, the opensensor can send some feedback to the phone when the temperature reaches a certain value. For the gateway approach we have created a simple protocol for communication, based on three tags:

- ACTION \{DISPLAY, SPEECH, SMS, MMS\}

- BODY $\{$ any $\}$

- KEYVALUe $\{$ any

When the opensensor needs to communicate with the phone, it sends a message formatted according to this protocol. When the phone receives the message, depending on the ACTION tag, it can perform different actions: display an alert on the screen, send an SMS or an MMS, or speak out the body of the message using the text2speech (functionality available on S60 phones.) An example of a typical message from the opensensor to the phone can be:

\section{$<$ ACTION $>$ SMS \\ $<$ BODY>Warning!!! Temperature $41 \mathrm{C}$ \\ $<$ KEYVALUE $>+123456789$}

On the phone side, an application is running in the background and waiting for information from the opensensor. In Figure 7 some screenshots of a testbed application we have developed are shown. In the retrieval approach, the phone

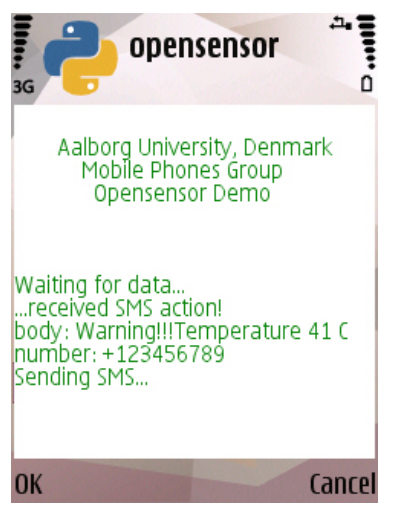

(a) The opensensor sends an SMS action command when the temperature exceeds a certain value.
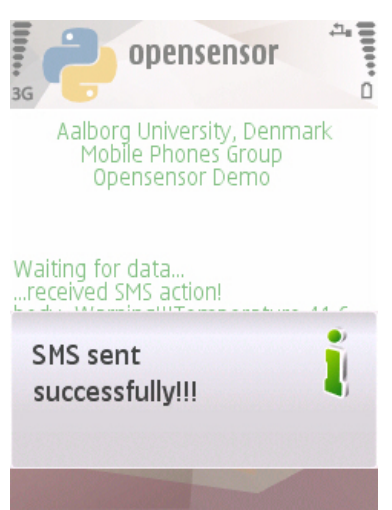

(b) The phone receives the SMS command and sends an SMS to a specified number.
Figure 7: Example of an application written in Python for S60 that allows the opensensor to use the mobile phone as a gateway to send SMS.

is using the capability of the sensors mounted on the board to retrieve information about the surroundings. The phone needs to run an application able to send some commands to the opensensor via Bluetooth when it needs to get the information. To program applications on mobile phones several programming languages are available. The choice highly depends on the platform, as described in [6]. In the Listing 1 a simple script written in Python for S60 [7], one possible choice for programming on Symbian OS platform, is shown. The script allows to open a Bluetooth socket communication with the opensensor and send data to it. This code example can be used to send ON/OFF commands to the opensensor, for example for switching the green LED on and off. 


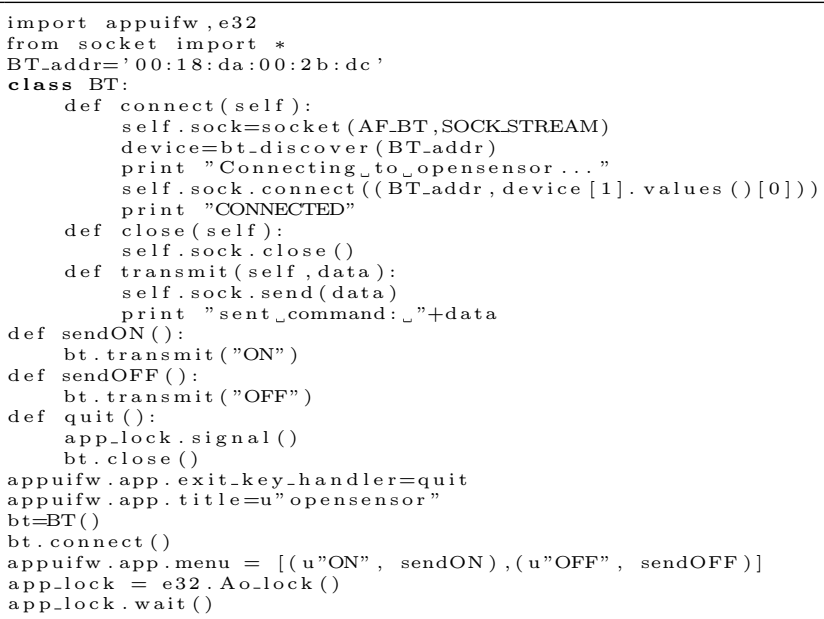

Listing 1: Code example of an application written in Python for S60 to send on/off commands to the opensensor.

To guarantee more portability among different mobile phone platforms, Java Mobile can be used for applications development. On the webpage [4] more code snippets for communication between the opensensor and mobile phones supporting Java Mobile can be found.

\section{5 opensensor educational support}

Besides the hardware and software also basic teaching material is freely available. The teaching material contains basic information about the opensensor and how to program on it. The material is available in PowerPoint and pdf format.

\section{OPENSENSOR PROJECTS}

In this section we describe shortly some of the projects carried out at the Aalborg University or the Technical University of Berlin.

\subsection{Parksensor}

The main idea is to move high priced functionality from the car to the mobile phone and spice those services even up. One example is the presented park sensor idea. A car is equipped with ultra sonic distance sensors which are connected wirelessly with the mobile phone. The mobile phone is placed within the car showing the distances to the nearest objects. Besides the display functionality, the mobile phone can support the driver with audio information (we are using text2speech functionalities of S60 phones). As the service is bundled to the phone it makes no sense to steal anything from the car. The parksensor is described in more detail in $[8]$.

\subsection{Mesh Sensor Networks}

The purpose of this project was to design and implement a medium access control (MAC) protocol for the ISM module of the opensensor platform, allowing direct communication among the opensensors and proving a solution to overcome possible collisions between packets during transmission. A collision avoidance scheme with acknowledgments and carrier sensing has been designed and implemented to minimize

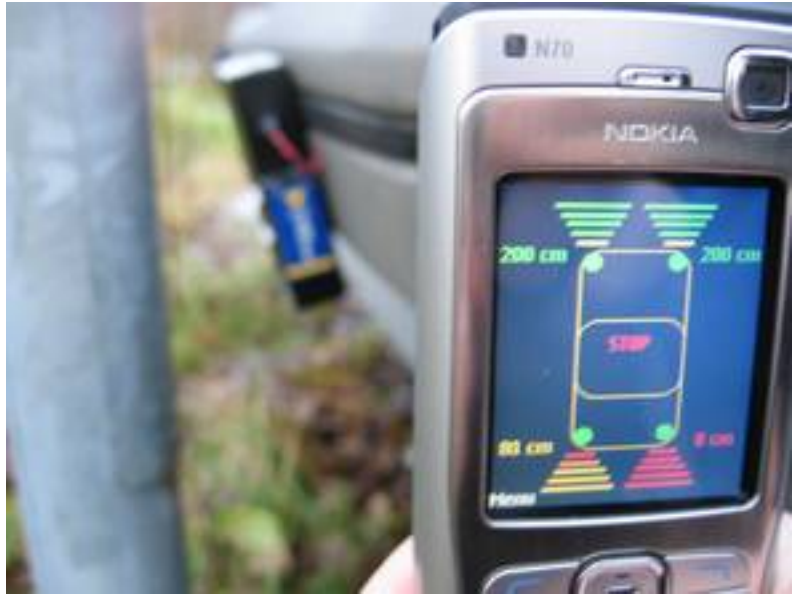

Figure 8: A picture taken during the tests of the parksensor application. On the screen of the phone, the colored bars indicate the distance measurements coming from the four sensors placed on the car.

data loss and delay, with a minimal number of retransmissions. To test the reliability of this wireless network and as an application proposal, two mobile phones are connected over a mesh network formed by opensensors. The opensensor uses the nRF905 device to communicate among each other, while the mobile phones uses Bluetooth to communicate with the opensensors. In the end it was possible to exchange information among the mobile phones over the mesh architecture.

\subsection{Distributed Storage}

Some applications introduce only partially connected sensor networks leading to lower reliability or availability of the measured data. The objective of this project is to develop and analyze a data distribution method which can increase the system reliability and keep the memory consumption low on each device. A Reed-Solomon coding scheme is applied as a solution where a certain number of unavailable devices can be tolerated without jeopardizing the system reliability. A probabilistic system model is derived to describe the distribution and reconstruction of a message from a sensor to the gateway. This model is verified and visualized by means of a simulation implemented in Java. A prototype of a system containing two data devices and one redundant device is implemented to test the model in a real life scenario. This prototype can be seen in Figure 9. Performance evaluation shows that the probability to receive all sensor measurements by using the proposed cooperative data distribution method is higher compared with the non-cooperative case.

\section{CONCLUSION}

This paper has introduced the opensensor platform, which follows the concept of open source. Besides the software, also the hardware design and the educational means are freely available. The opensensor can be used for pure wireless sensor network concepts or, as used by the others, for the convergence of wireless sensor networks with mobile phones. Throughout the text, we have motivated the convergence of mobile phones and wireless sensor networks to create better 


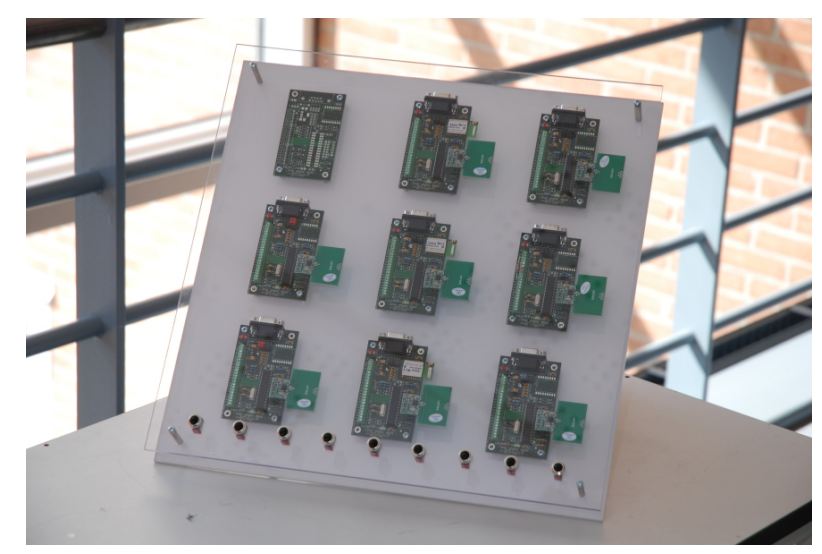

Figure 9: The prototype implementation of the Distributed Storage project in a network of opensensors.

and more innovative services for the user. This convergence will take place by embedded and external wireless sensors and mobile phones.

\section{ACKNOWLEDGEMENT}

We would like to thank our students of AAU and TUB that have contributed to the opensensor platform in their student projects or in the lectures.

\section{REFERENCES}

[1] Mobile Phone Programming and its Application to Wireless Networking. Springer, 2007, ch. Using In-built RFID/NFC, Cameras, and 3D Accelerometers as Mobile Phone Sensors.

[2] C. S. Jensen and D. Tiesyte, "Gps data management with applications in collective transport," http://www.cs.aau.dk/TransDB/papers/its.pdf.

[3] mobiledevices.kom.aau.dk.

[4] mobiledevices.kom.aau.dk/opensensor.

[5] S. Rein, C. Gühmann, and F. Fitzek, Mobile Phone Programming and its Application to Wireless Networking. Springer, 2007, ch. Sensor Networks for Distributed Computing.

[6] F. Fitzek and F. Reichert, Eds., Mobile Phone Programming and its Application to Wireless Networking. Springer, 2007.

[7] J. Scheible and V. Tuulos, Mobile Python: Rapid Prototyping of Applications on the Mobile Platform. Wiley, ISBN: 978-0-470-51505-1, 2007.

[8] J. Rasmussen, P. Østergaard, J. Jensen, A. Grauballe, G. Perrucci, B. Krøyer, and F. Fitzek, Mobile Phone Programming and its Application to Wireless Networking. Springer, 2007, ch. Parking Assistant Application. 